\title{
Call for Papers! Special Issue on Models of Discovery: Mining the Scientific Literature
}

Vol. 2 No. 2, 2017 p 96

DOI: 10.1515/jdis-2017-0010

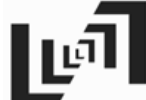

JDIS

Journal of Data and Information Science
The Journal of Data and Information Science (JDIS) is honored to invite Professors Neil R. Smalheiser and Aaron M. Cohen to be Guest Editors for the Special Issue on Models of Discovery: Mining the Scientific Literature.

We call for papers that employ innovative conceptual or methodological approaches to discovering new knowledge, using the scientific literature as the source. Possible topics include:

- Characterizing the core $v s$. the periphery of a discipline;

- Identifying research areas that are particularly promising or particularly neglected;

- Machine learning, including graph-based, tensor-based and deep learning approaches, active learning, merging heterogeneous textual and biological data, etc.;

- Applying advanced Natural Language Processing (NLP) such as sentiment analysis, argumentation analysis, or recognizing humor and sarcasm;

- Use of outside information to guide discovery, such as bibliometrics, distant supervision, or cross-corpus information;

- Use of implicit information to guide discovery;

- Evidence synthesis and summarization techniques to guide discovery;

- Identifying findings that have low credibility or are unlikely to be true.

All manuscripts submitted to the special issue will be reviewed in accordance with standard journal procedures and be finally evaluated by the Issue Editors and Co-EICs.

All manuscripts should be submitted via JDIS's online manuscript processing system: https://mc03.manuscriptcentral.com/jdis, and state "Special Issue on Models of Discovery: Mining the Scientific Literature" in the cover letter.

\section{Issue Editors:}

Prof. Neil R. Smalheiser Prof. Aaron M. Cohen

University of Illinois at Chicago, USA Oregon Health \& Science University, USA

Email: neils@uic.edu

E-mail: cohenaa@ohsu.edu

\section{Important dates:}

Expected publication date: Sept. 1, 2017

Deadline for submission: Jul. 1, 2017

If you have any suggestions or questions about the special issue, please feel free to contact us at liufh@mail.las.ac.cn or jdis@mail.las.ac.cn. 